Pesq. Vet. Bras. 37(2):195-203, fevereiro 2017 DOI: 10.1590/S0100-736X2017000200015

\title{
Perfil metabólico de vacas Jersey clinicamente saudáveis ${ }^{1}$
}

\author{
Paula B. de Alvarenga ${ }^{2}$, Amanda L. Rezende ${ }^{2}$, Felipe B. Justo², Soraia R. Rezende², \\ João C.G. Cesar ${ }^{2}$, Ricarda M. Santos ${ }^{2}$, Antonio V. Mundim² e João P.E. Saut ${ }^{2 *}$
}

\begin{abstract}
Alvarenga P.B., Rezende A.L., Justo F.B., Rezende S.R., Cesar J.C.G., Santos, R.M., Mundim A.V. \& Saut J.P.E. 2017. [Metabolic profile of clinically healthy Jersey cows.] Perfil metabólico de vacas Jersey clinicamente saudáveis. Pesquisa Veterinária Brasileira 37(2):195-203. Laboratório de Saúde em Grandes Animais, Faculdade de Medicina Veterinária, Universidade Federal de Uberlândia, Av. Pará 1720, Bloco 2T, Campus Umuarama, Bairro Umuarama, Uberlândia, MG 38400-902, Brazil. E-mail: jpsaut@ufu.br

The understanding of animal metabolism is important for the success of livestock activity. The aim of this study was to determine the serum biochemical profile of clinically healthy Jersey cows in pre and postpartum, housed in semi-intensive production system in Uberlândia, Minas Gerais, Brazil. Clinical examination and blood collection from 40 multiparous lactating dairy cows, between -150 days prepartum up to 60 days postpartum (DPP) were performed. At the laboratory, serum parameters of total proteins, albumin, globulins, urea, creatinine, non-esterified fatty acids (NEFA), $\beta$-hydroxybutyrate (BHBA), triglycerides, cholesterol, high density lipoprotein (HDL), very low density lipoproteins (VLDL), low density lipoproteins (LDL), aspartate aminotransferase (AST), gamma-glutamyl transferase (GGT), creatine kinase (CK), calcium, phosphorus and magnesium were analyzed. To the total of cows $(n=40)$ enrolled, only 21 cows were complied with the inclusion criterias. It was proceeded the Tukey test for parametric data, and to the nonparametric data it was held the Kruskal Wallis test for the comparison between days evaluated. There was no interference of negative energy balance and subclinical hypocalcemia in the occurrence of uterine diseases and reproductive performance. In conclusion, Jersey cows have biochemical profile characterized by hyperglobulinemia during peripartum, and high levels of triglycerides, but without impaired liver function and reproductive performance.
\end{abstract}

INDEX TERMS: Jersey cows, dairy cows, metabolic profile, postpartum, transition period.

RESUMO.- 0 conhecimento do metabolismo dos animais é de suma importância para se obter sucesso em qualquer atividade que envolva rebanhos. Objetivou-se determinar o perfil bioquímico sérico de vacas Jersey clinicamente saudáveis no pré e pós-parto, mantidas em sistema de criação semi-intensivo na região de Uberlândia, Minas Gerais, Brasil. Realizou-se o exame clínico e coleta de sangue de 40 vacas da raça Jersey multíparas e lactantes em 15 momentos, entre -150 dias pré-parto até 60 dias pós-parto (DPP). No laboratório, foram analisadas as concentrações séricas de pro-

\footnotetext{
${ }^{1}$ Recebido em 16 de fevereiro de 2016.

Aceito para publicação em 4 de setembro de 2016.

Parte de Dissertação de Mestrado do primeiro autor, com apoio CAPES e FAPEMIG.

${ }^{2}$ Laboratório de Saúde em Grandes Animais, Faculdade de Medicina Veterinária, Universidade Federal de Uberlândia (UFU), Av. Pará 1720, Bloco 2T, Campus Umuarama, Bairro Umuarama, Uberlândia, MG 38400-902, Brasil.*Autor para correspondência: jpsaut@ufu.br
}

teínas totais, albumina, globulinas, ureia, creatinina, ácidos graxos não esterificados (NEFA), $\beta$-hidroxibutirato (BHBA), triglicerídeos, colesterol, lipoproteínas de alta densidade (HDL), lipoproteínas de muita baixa densidade (VLDL), lipoproteínas de baixa densidade (LDL), aspartato aminotransferase (AST), gama-glutamil transferase (GGT), creatina quinase (CK), cálcio, fósforo e magnésio. Dos 40 animais, apenas 21 vacas atenderam aos critérios de inclusão e seus dados foram utilizados. Procedeu-se o teste de Tukey para os dados paramétricos e para os dados não paramétricos realizou-se o teste de Kruskal Wallis na comparação entre os momentos avaliados. Não houve interferência do balanço energético negativo e da hipocalcemia subclínica na ocorrência de doenças uterinas e no desempenho reprodutivo. Concluiu-se que vacas da raça Jersey apresentam perfil bioquímico caracterizado por hiperglobulinemia durante o periparto, além de níveis elevados de triglicérides, entretanto, sem comprometimento da função hepática e desempenho reprodutivo. 
TERMOS DE INDEXAÇÃO: Vacas Jersey, perfil metabólico, período de transição, pós-parto, vacas leiteiras.

\section{INTRODUÇÃO}

A composição bioquímica do plasma sanguíneo retrata a situação metabólica dos tecidos dos animais, permitindo inferências a respeito do funcionamento de seu organismo, bem como da adaptação do indivíduo no ambiente em que está inserido (González \& Silva 2006). Vacas no periparto passam por estresse fisiológico que promove alterações metabólicas significativas, que podem ser de ordem proteica, mineral, enzimática ou energética, que por sua vez, refletem na saúde e produtividade do animal (González \& Scheffer 2002, Chapinal et al. 2011, Oliveira et al. 2014).

Para que os animais voltem a conceber após a parição é preciso que ocorram alguns eventos no pós-parto imediato como a eliminação da placenta, involução uterina, regeneração do endométrio, eliminação bacteriana e retorno ovariano à ciclicidade (Sheldon 2004, Sheldon et al. 2008). Dessa forma, para lograr bons índices sanitários e reprodutivos do rebanho, é importante conhecer o padrão fisiológico do metabolismo da raça em questão, para assim, poder diagnosticar precocemente eventuais desordens e intervir de maneira a saná-las. 0 perfil metabólico sofre interferência de fatores como raça, idade, nível de produção, clima, estado fisiológico (lactação, gestação, etc.), dieta e manejo dentre outros fatores (Peixoto et al. 2010, González et al. 2014).

Conhecer o metabolismo animal permite diagnosticar doenças precocemente, evitando assim possíveis perdas econômicas devido aos custos com tratamento, dias não produtivos que o animal doente passa a somar para a propriedade e, eventualmente, o descarte involuntário do animal (Martins et al. 2013).

Em vacas Holandesas, criadas na América do Norte e Europa, a bioquímica sérica tem sido intensamente empregada como ferramenta na prevenção de doenças no periparto (Dubuc et al. 2010, Chapinal et al. 2011, Chapinal et al. 2012, Roberts et al. 2012). Entretanto, em vacas Jersey, especialmente no Brasil, ainda não existem trabalhos que apresentem o perfil metabólico sérico normal de vacas em produção e nem correlacionando com doenças e desempenho reprodutivo pós-parto. Isso caracteriza um fator importante a ser considerado, haja vista que se trata de uma raça com alto potencial produtivo, e que vem ganhando espaço na pecuária leiteira, em função da crescente busca por redução do custo de produção, uma vez que a raça é caracterizada por ingestão de alimentos inferior e por apresentar percentual de sólidos totais no leite superior a outras raças (Aikman et al. 2008, Prendiville et al. 2009).

Ao se considerar que os sistemas de produção empregados têm se tornado cada vez mais eficientes, visto o alto potencial produtivo dos animais e o emprego de novas tecnologias de produção, o risco de transtornos metabólicos aumenta, predispondo os animais a infecções uterinas pós-parto e a baixa eficiência reprodutiva, podendo trazer sérios prejuízos. Desta forma, objetivou-se determinar o perfil bioquímico sérico de vacas Jersey, criadas em condições de clima e manejo locais, que não apresentassem en- fermidades pré-parto nem puerperais, entre 150 dias antes do parto até os 60 dias pós-parto, no intuito de auxiliar na formação de valores de referência para a raça Jersey.

\section{MATERIAL E MÉTODOS}

Animais, local e manejo. 0 projeto foi aprovado pela Comissão de Ética no Uso de Animais (CEUA) da Universidade Federal de Uberlândia, sob o protocolo 013/15. Foram utilizadas 21 vacas Jersey, adultas, lactantes, multíparas, do total de 40 animais inicialmente acompanhados, de uma propriedade localizada no $\mathrm{Mu}-$ nicípio de Uberlândia, Minas Gerais, com produção média diária de $20 \mathrm{~kg}$ leite, no período de janeiro a dezembro de 2015.

0 município de Uberlândia se localiza na mesorregião do Triângulo Mineiro e Alto Paranaíba, Estado de Minas Gerais, Sudeste do Brasil, entre as coordenadas $19^{\circ} 14^{\prime}$ e $18^{\circ} 34^{\prime}$ de latitude Sul e $48^{\circ} 50^{\prime}$ e $47^{\circ} 03^{\prime}$ de longitude a Oeste de Greenwich (Santos \& Petronzio 2011). Sua altitude média é de 863 metros e o clima da região, segundo a classificação de Köppen, do tipo Aw, megatérmico com chuvas concentradas no verão (dezembro a março) e secas no inverno (junho a setembro) (Giffoni \& Rosa 2007). No período avaliado, a Estação climatológica do Instituto de Geografia da Universidade Federal de Uberlândia registrou temperatura média de $20,72 \pm 6,97^{\circ} \mathrm{C}$, umidade relativa do ar de $55,26 \pm 26,11 \%$ e precipitação média de $103 \pm 91,4 \mathrm{~mm}$.

0 manejo das vacas em lactação foi composto de duas ordenhas diárias, sendo os animais divididos em cinco lotes, de acordo com a ordem de parição, produção leiteira e ocorrência de mastites. A média dos dias em lactação (DEL) das vacas, no momento de inserção no experimento (d-150), foi de $212 \pm 32,97$ dias. A secagem e/ou tratamento da mastite foi realizado com antibiótico intramamário à base de cefalexina, neomicina ou ampicilina e cloratina.

Durante todo o ano foram mantidos em pastagens irrigadas de Brachiaria decumbens e Tifton, adubadas ao final de cada ciclo de pastejo baseado na altura do dossel forrageiro. No período seco do ano, a dieta foi complementada com 60,4\% de resíduo úmido de cervejaria (cevada) para vacas em lactação, $6 \%$ de farelo de soja, $12,1 \%$ de milho reidratado, $1,2 \%$ premix capim e $0,3 \%$ consistiram em mineralização e ração comercial Cooprata $24 \%{ }^{\circledR}$ (Prata, MG, Brasil) ao cocho durante o manejo de ordenha, na proporção de $1 \mathrm{Kg}$ de concentrado para cada $3 \mathrm{Kg}$ de leite produzidos. No verão, o rebanho foi mantido a pasto, e recebeu complementação alimentar com concentrado comercial contendo $24 \%$ de proteína, $2,82 \%$ de extrato etéreo, $11,27 \%$ de matéria fibrosa, $8,45 \%$ de fibra em detergente ácido (FDA) e 12,68\% de matéria mineral, na proporção de $1 \mathrm{~kg}$ de concentrado para cada $3 \mathrm{~kg}$ de leite produzidos.

Delineamento experimental. Os momentos estabelecidos para avaliação clínica e coleta de dados foram: 150, 120, 90, 60, $28,21,14,7$ e 2 dias pré-parto (d-150, d-120, d-90, d-60, d-28, d-21, d-14, d-7, d-2), e após o parto aos 2, 7, 14, 21, 28 e 60 dias pós-parto (DPP). Em cada momento eram realizados os seguintes procedimentos: a) avaliação do escore de condição corporal (ECC), b) exame clínico e c) coleta de sangue. O ECC foi avaliado sempre pelo mesmo observador, variando de um a cinco com subunidades de 0,25, como descrito por Edmonson et al. (1989).

0 exame clínico dos animais foi procedido segundo Feitosa (2014) e utilizado como critério de permanência ou exclusão dos animais no estudo. Além do exame clínico, atenção especial foi dada ao sistema genital feminino, em relação à involução uterina e presença de infecções uterinas diagnosticadas, conforme Oliveira et al. (2014).

0 exame físico específico do sistema genital feminino foi realizado com auxílio da ultrassonografia transretal com aparelho 
DP 2200vet (Mindray ${ }^{\circledR}$ ) e transdutor eletrônico 5-10 MHz, com avaliação da secreção vaginal por meio do equipamento de coleta "metricheck", conforme Williams et al. (2005) aos 28 DPP. Neste momento também foi feita a citologia endometrial para o diagnóstico da endometrite citológica pela técnica de esfregaço endometrial (cytobrush) conforme descrito por Kaufmann et al. (2009), sendo considerada contagem de neutrófilos superior a 10\% como indicativo de endometrite citológica (Gilbert et al. 2005, Kasimanickam et al. 2005).

As alterações metabólicas e/ou doenças puerperais foram diagnosticadas da seguinte maneira:

Retenção de placenta: diagnosticada de acordo com Williams et al. (2005) e Sheldon et al. (2009), quando não havia eliminado as membranas fetais em até 12 horas transcorridas do parto (Ferreira 2010, Rezende 2013).

Metrite: diagnosticada até os $21 \mathrm{DPP}$, pelo aumento de volume uterino, sendo classificada em leve (útero aumentado de volume, associado à descarga uterina achocolatada purulenta, apresentando ou não odor fétido, sem a presença de sinais sistêmicos), moderada (quando além dos sinais citados anteriormente havia hipertermia, queda na produção leiteira e apatia) e grave (presença de toxemia, inapetência e depressão) de acordo com o proposto por Sheldon et al. (2009) e Williams et al. (2005).

Endometrite clínica: conforme Williams et al. (2005) e Sheldon et al. (2009), porém utilizando apenas a avaliação aos 28 DPP, quando da presença de descarga vaginal mucopurulenta.

Hipocalcemia: quando em até 72 horas pós-parto apresentavam paresia de membros posteriores com dificuldade para se levantarem ou mesmo não conseguiam se levantar (González et al. 2014).

Hipocalcemia subclínica: determinada com base nos níveis séricos de cálcio inferiores a $8,4 \mathrm{mg} / \mathrm{dL}$, conforme descrito por Chapinal et al. (2011) e Chapinal et al. (2012).

Balanço energético negativo: diagnosticado, no pré-parto, pelas concentrações de NEFA superiores a $2,7 \mathrm{mEq} / \mathrm{L}$ e no pós parto, pelos níveis de NEFA superiores a 7,2mEq/L e BHBA superior a 1,0mg/dL, conforme descrito por Ospina et al. (2010).

Coleta e processamento de material. As amostras de sangue, tal qual descrito por Oliveira et al. (2014), foram colhidas por punção da veia caudal mediana em tubos próprios de $9 \mathrm{~mL}$, estéreis, a vácuo contendo gel separador e ativador de coágulo (Vacuplas $\mathrm{t}^{\circledR}$ ) para se proceder as análises bioquímicas. Estas foram transportadas em caixa isotérmica refrigerada ao Laboratório de Análises Clínicas do Hospital Veterinário (HV-UFU), centrifugadas a $720 \mathrm{~g}$ por 10 minutos em centrífuga sorológica INBRAS ${ }^{\circledR}$ para a obtenção do soro sanguíneo, que foi transferido à microtubos de $2 \mathrm{~mL}$ do tipo Eppendor ${ }^{\circledR} \mathrm{e}$ armazenado a $-20^{\circ} \mathrm{C}$ para posterior análise, não ultrapassando cinco dias pós-coleta. Ressalta-se que as coletas foram feitas sempre no período da manhã (entre 8 e 10 horas) logo após as atividades da primeira ordenha do dia.

As análises bioquímicas foram processadas em analisador automático multicanal ChemWell (Awareness Technology Inc. ${ }^{\circledR}$ ) a $37^{\circ} \mathrm{C}$, previamente calibrado (Calibra $\mathrm{H}$ ) e aferido com soro controle (Qualitrol 1). Os parâmetros bioquímicos séricos analisados por meio de kits diagnósticos Randox ${ }^{\circledR}$ e Labtest $^{\circledR}$ disponíveis no mercado foram: proteínas totais, albumina, globulinas, relação albumina/globulina, ureia, creatinina, ácidos graxos não esterificados (NEFA), $\beta$-hidroxibutirato (BHBA), triglicerídeos, colesterol, lipoproteína de alta densidade (HDL), aspartato aminotransferase (AST), gama-glutamil transferase (GGT), creatina quinase (CK), cálcio, fósforo, magnésio e relação cálcio/fósforo. Utilizando a equação de Friedewald et al. (1972) foram calculadas as concentrações das lipoproteínas de muita baixa densidade (VLDL) - triglicerídeos/5 e lipoproteínas de baixa densidade (LDL) - colesterol total-(HDL+VLDL).
Análise estatística. Os dados foram tabulados em planilhas do programa Microsoft Excel e posteriormente analisados pelo programa estatístico Graphpad Prism $5^{\circledR}$ (GraphPad Software Inc., La Jolla, CA). Os dados foram apresentados nos gráficos, por meio de média e erro padrão da média, e no quadro os intervalos de confiança. Para avaliação do comportamento das variáveis bioquímicas no intervalo avaliado, foi utilizado o teste de Kolmogorov-Smirnov para testar a normalidade dos dados e, a partir de então, aquelas constatadas como paramétricas foram analisadas segundo o teste de Tukey, enquanto os dados não paramétricos foram analisados pelo teste de Kruskal-Wallis, adotando como hipótese nula, que as médias dos dias de avaliação das variáveis fossem iguais, sendo, portanto, a hipótese $\mathrm{H}_{1}$ de que as médias fossem diferentes entre si. Para todas as análises realizadas considerou-se significância de $5 \%$.

\section{RESULTADOS}

Dos 40 animais inicialmente acompanhados, nove foram eliminados por descarte voluntário devido a enfermidades ocorridas no pré-parto e, outros dez no pós-parto por retenção de placenta $(n=4 / 10)$, metrite $(n=1 / 10)$, endometrite clínica $(n=2 / 10)$ e citológica $(n=9 / 10)$. Com isso, a análise das variáveis séricas contemplou apenas os 21 animais que não apresentaram nenhuma alteração clínica, sendo, portanto considerados animais clinicamente saudáveis.

Em relação às proteínas e metabólitos proteicos (Fig.1), observaram-se níveis séricos de proteínas totais superiores aos valores de referência apenas no período anterior ao periparto. A concentração sérica de albumina oscilou nos níveis mínimos de referência, apresentando valores inferiores a estes entre d-60 $(2,88 \pm 0,4 \mathrm{~g} / \mathrm{dL})$ e 2 DPP $(2,78 \pm 0,46 \mathrm{~g} / \mathrm{dL})$. Os baixos níveis de albumina e altos de globulinas fizeram com que a relação albumina/globulinas ficasse abaixo do esperado, entre 0,8 e 0,9 (Smith 2009), variando de $0,40 \pm 0,05$ a $0,69 \pm 0,3$. Os níveis de ureia oscilaram de $26,02 \pm 12,94$ a $48,61 \pm 15,31 \mathrm{mg} / \mathrm{dL}$, enquanto a concentração sérica de creatinina variou de $0,57 \pm 0,13$ a $0,85 \pm 0,18 \mathrm{mg} / \mathrm{dL}$.

Os valores referentes ao perfil bioquímico das enzimas hepáticas estão representados na Figura 2, não havendo variação das enzimas AST e GGT. As vacas Jersey apresentaram o perfil bioquímico hepático de AST $(73,05 \pm 22,16$ a $102,41 \pm 27,51 \mathrm{UI} / \mathrm{L})$ e GGT $(16,15 \pm 5,45$ a $20,94 \pm 9,93$ UI/L) dentro dos valores de referência. Já os níveis de CK foram superiores aos de referência desde d-120 até d-7, e entre 7 DPP e 14 DPP, variando de $229,86 \pm 131,27$ a $734,28 \pm 1151,15 \mathrm{UI} / \mathrm{L}$.

Os níveis de colesterol reduziram gradativamente durante o pré-parto, de $136 \pm 37,99 \mathrm{mg} / \mathrm{dL}$ para $68 \pm 25,45 \mathrm{mg} /$ $\mathrm{dL}$. Após, assumiram uma curva ascendente no puerpério, variando de $75,4 \pm 18,65 \mathrm{mg} / \mathrm{dL}$ (2DPP) a $140 \pm 23,99 \mathrm{mg} / \mathrm{dL}$ (60 DPP), quando ultrapassaram o limiar máximo de referência $(80-120 \mathrm{mg} / \mathrm{dL})$. Em relação aos triglicerídeos, as concentrações estiveram acima dos níveis basais normais (0-14mg/dL), exceto aos 7 DPP, quando a concentração média foi de 13,12 $\pm 6,87 \mathrm{mg} / \mathrm{dL}$. As lipoproteínas HDL seguiram padrão semelhante ao observado para o colesterol, apresentando seus níveis mais baixos no período de d-28 a d-2 (Fig.3). 

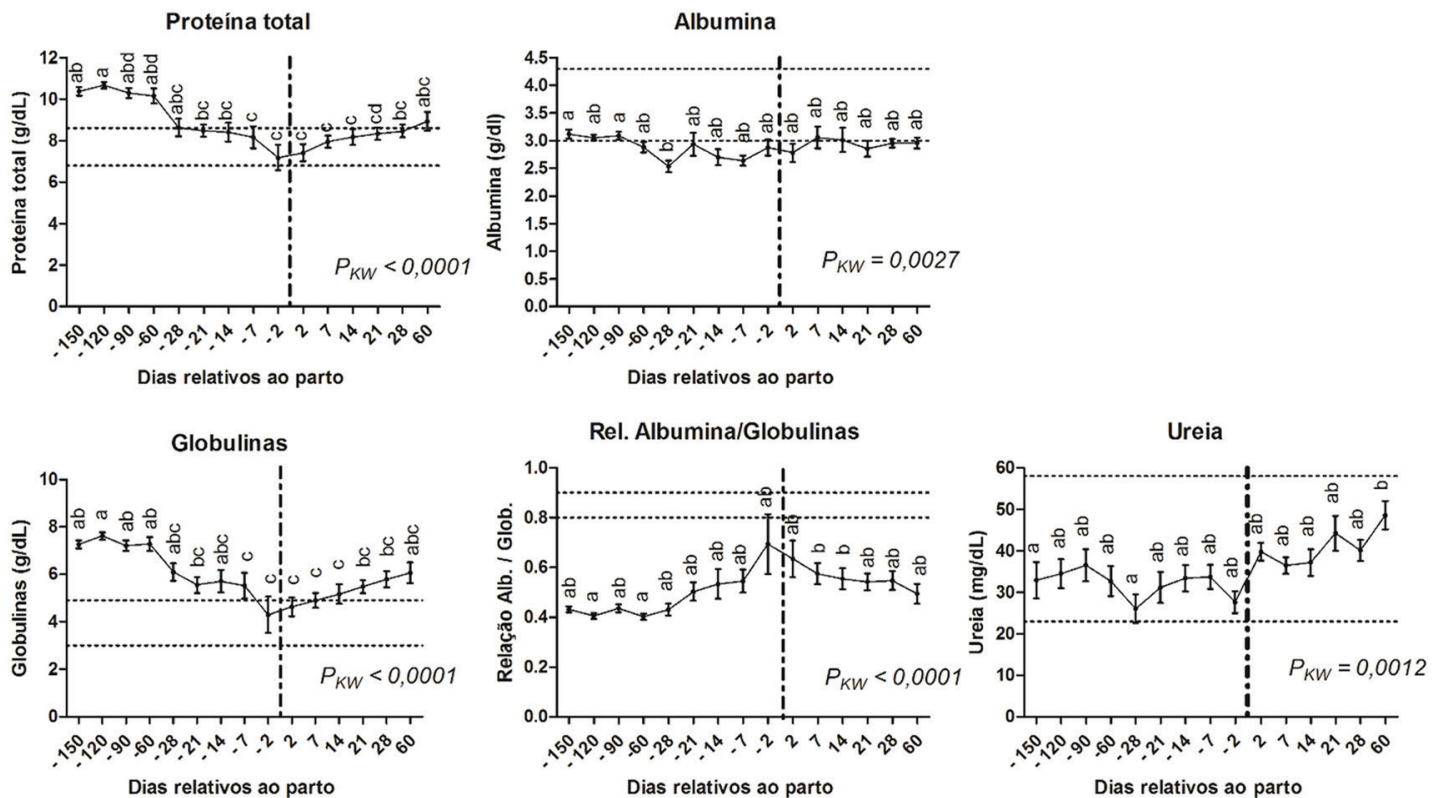

Fig.1. Proteínas e metabólitos proteicos de vacas Jersey multíparas clinicamente sadias, criadas em sistema semi-intensivo na região de Uberlândia, MG. (Nota: Letras diferentes entre os dias de avaliação indicam diferença estatística $(\mathrm{P}<0,05)$ pelo teste de Kruskall Wallis $\left(P_{\mathrm{KW}}\right)$. Vacas multíparas com produção média de $20 \mathrm{~kg}$ leite/dia. Linhas tracejadas representam os limites dos valores de referência adotados conforme Smith (2009) para Proteínas totais (6,8-8,6g/dL), Albumina (3-4,3g/dL), Globulinas (3-4,9g/dL), Relação a/g (0,8-0,9g/dL) e Creatinina (0,9-1,3mg/dL) e segundo David Eckersall (2008) para ureia (23-58mg/dL).)
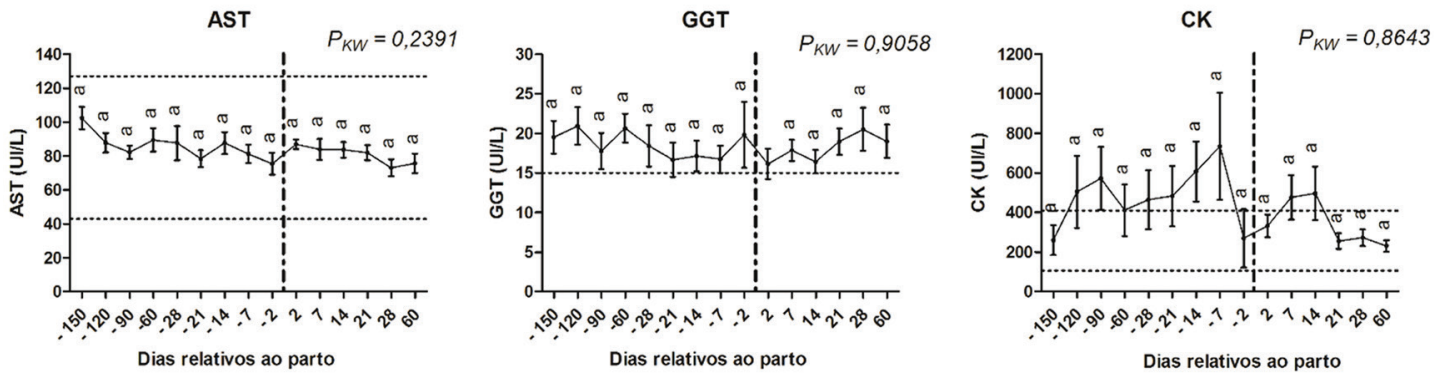

Fig.2. Perfil bioquímico sérico de enzimas de vacas Jersey multíparas clinicamente sadias, criadas em sistema semi-intensivo na região de Uberlândia, MG. (Nota: Letras diferentes entre os dias de avaliação indicam diferença estatística $(P<0,05)$ pelo teste de Kruskall Wallis $\left(P_{\mathrm{KW}}\right)$. Vacas multíparas com produção média de $20 \mathrm{~kg}$ leite/dia. Linhas tracejadas representam os limites dos valores de referência adotados conforme Smith (2009) para AST (43-127 UI/L), GGT (15-39 UI/L) e CK (105-409 UI/L).)

Durante o pré-parto, os valores encontrados para os ácidos graxos não esterificados (NEFA) estiveram dentro dos valores de referência adotados $(\leq 0,27 \mathrm{mEq} / \mathrm{L})$ apenas aos $\mathrm{d}-150(0,24 \pm 0,14 \mathrm{mg} / \mathrm{dL}), \mathrm{d}-21(0,20 \pm 0,14 \mathrm{mg} / \mathrm{dL})$ e $\mathrm{d}-14$ $(0,26 \pm 0,19 \mathrm{mg} / \mathrm{dL})$. Já no puerpério, suas concentrações se mantiveram dentro dos níveis adotados $(\leq 0,72 \mathrm{mEq} / \mathrm{L})$, no entanto, apresentou um pico no segundo dia de puerpério $(0,61 \pm 0,51 \mathrm{mEq} / \mathrm{L})$ com uma queda significativa até os 60 DPP $(0,16 \pm 0,1 \mathrm{mEq} / \mathrm{L})$ (Fig.4).

A análise de corpos cetônicos permitiu inferir que as vacas Jersey com puerpério fisiológico apresentam níveis de BHBA aceitáveis para o puerpério $(\leq 1,0 \mathrm{mmol} / \mathrm{L})$ (Ospina et al. 2010) em todo o período avaliado (Fig.4). Verificou-se o maior valor no período entre d-150 $(0,59 \pm 0,28 \mathrm{mmol} / \mathrm{L})$ e d-90 $(0,47 \pm 0,13 \mathrm{mmol} / \mathrm{L})$, enquanto o menor valor foi observado aos 7 DPP $(0,19 \pm 0,21 \mathrm{mmol} / \mathrm{L})$. 0 ECC médio observado foi de 3,1 no pré-parto e 2,7 no pós-parto.

$\mathrm{Na}$ avaliação do perfil bioquímico sérico mineral, observou-se que as concentrações médias de cálcio, fósforo e magnésio não diferiram muito dos padrões adotados como normais para vacas leiteiras em fases e condições semelhantes (Fig.5).

Os níveis séricos de cálcio no pré-parto atenderam aos valores de referência propostos para vacas Holandesas (9,7-12,4mg/dL) (Rucker et al. 2008), porém no periparto imediato (d-2 a 2 DPP) estavam reduzidos. A partir do 210 dia de puerpério, a concentração sérica desse mineral foi reestabelecida, porém, não ultrapassando o limite de $8,4 \mathrm{mg} / \mathrm{dL}$ considerado como referência para hipocalcemia subclínica (Fig.5). Verificou-se que 80,9\% $(17 / 21)$ das vacas apresentaram no periparto níveis reduzidos de cálcio $(<8,4 \mathrm{mg} / \mathrm{dL})$, sendo que $57,1 \%(12 / 21)$ ocorreram no pré-parto e $76,2 \%(16 / 21)$ no pós-parto. Entre 2 e 21 DPP, as concentrações de cálcio observadas foram, respectivamente, $7,4 \pm 1,74 \mathrm{mg} / \mathrm{dL}, 8,08 \pm 1,24 \mathrm{md} /$ $\mathrm{dL}, 8,16 \pm 1,34 \mathrm{mg} / \mathrm{dL}$ e $7,25 \pm 2,53 \mathrm{mg} / \mathrm{dL}$. Por outro lado, a maior concentração desse mineral ocorreu em d-150 $(10,36 \pm 1,3 \mathrm{mg} / \mathrm{dL})$. 
Os teores séricos de fósforo permaneceram abaixo dos limites de referência (5,6-6,5mg/dL) no período de d-90 a 14 DPP, apresentando-se dentro dos valores normais apenas entre d-150 e d-120 e após os 21 DPP. 0 elemento magnésio oscilou $(2,17 \pm 0,47$ a $2,57 \pm 0,75 \mathrm{mg} / \mathrm{dL})$ próximo ao limite máximo de referência $(1,8-2,3 \mathrm{mg} / \mathrm{dL})$, não apresentando diferença durante todo o período avaliado (Fig.5).

Os intervalos de confiança para todos os elementos avaliados do perfil metabólico estão apresentados no Quadro 1, com os valores mínimos e máximos na fase do periparto, três semanas antes até três semanas do pós-parto.

Os índices reprodutivos dos animais que permaneceram no experimento $(n=21)$ podem ser visualizados no Quadro 2.

\section{DISCUSSÃO}

Notou-se na literatura consultada uma carência de dados do perfil metabólico específico para a raça Jersey e, principalmente, que contemplassem a avaliação clínica cons- tante, com foco especial às doenças metabólicas e sistema genital feminino no pós-parto. Além disso, que apresentasse o desempenho reprodutivo destes animais clinicamente saudáveis após a parição.

0 delineamento proposto nesta pesquisa permite que os resultados possam auxiliar na formação de valores de referência específicos para esta raça, criada em regiões tropicais, pois se percebeu que muitos elementos não estavam dentro dos valores de referência das literaturas consagradas (Smith 2009), além de apresentarem variações de acordo com as fases produtivas.

0 perfil sérico proteico das vacas Jersey clinicamente saudáveis caracterizou-se no pré-parto por hiperproteinemia devido à hiperglobulinemia e normoalbuminemia, embora esta última oscilando nos valores mínimos de referência de 3 a 4,3g/dL, segundo Smith (2009). Este quadro alterou-se apenas próximo ao parto, entre d-2 e 7 DPP, com normoproteinemia, normoglobulinemia e hipoalbuminemia. Esta hiperproteinemia por hiperglobulinemia,

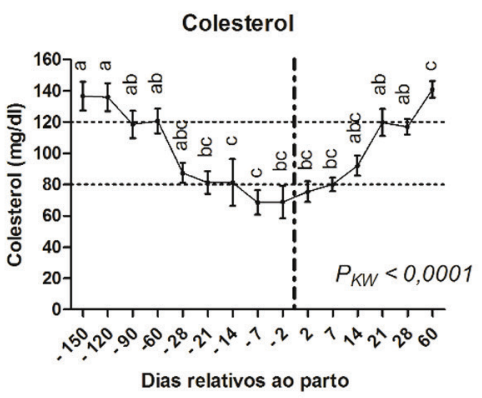

HDL

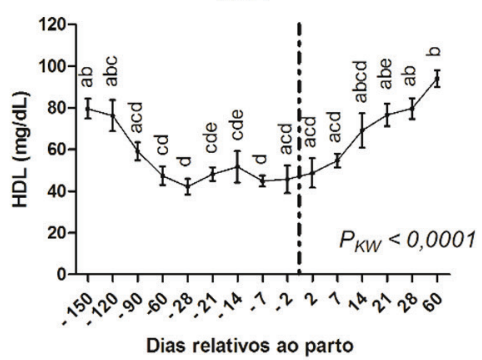

Triglicérides

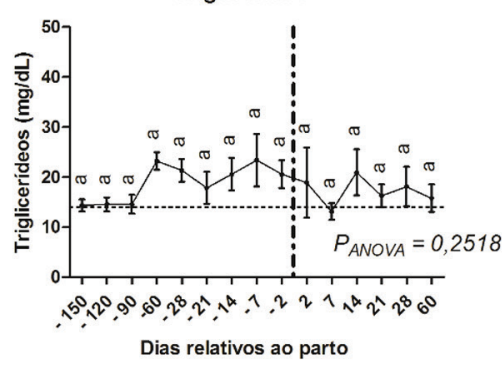

VLDL

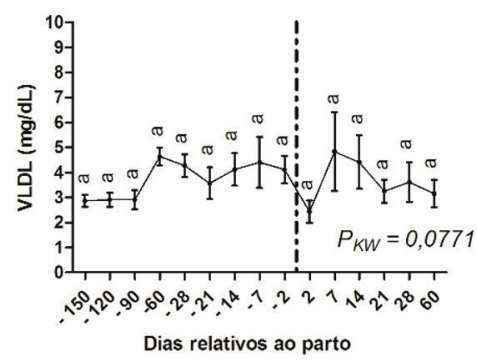

Fig.3. Indicadores do balanço energético negativo (BEN) de vacas Jersey multíparas clinicamente sadias, criadas em sistema semi-intensivo na região de Uberlândia, MG. (Nota: Letras diferentes entre os dias de avaliação indicam diferença estatística $(\mathrm{P}<0,05)$ pelo teste de Kruskall Wallis $\left(P_{\mathrm{KW}}\right)$. Vacas multíparas com produção média de $20 \mathrm{~kg}$ leite/dia. Linhas tracejadas representam os limites dos valores de referência adotados conforme Smith (2009) para Colesterol (80-120mg/dL) e Triglicérides (0-14mg/dL).)
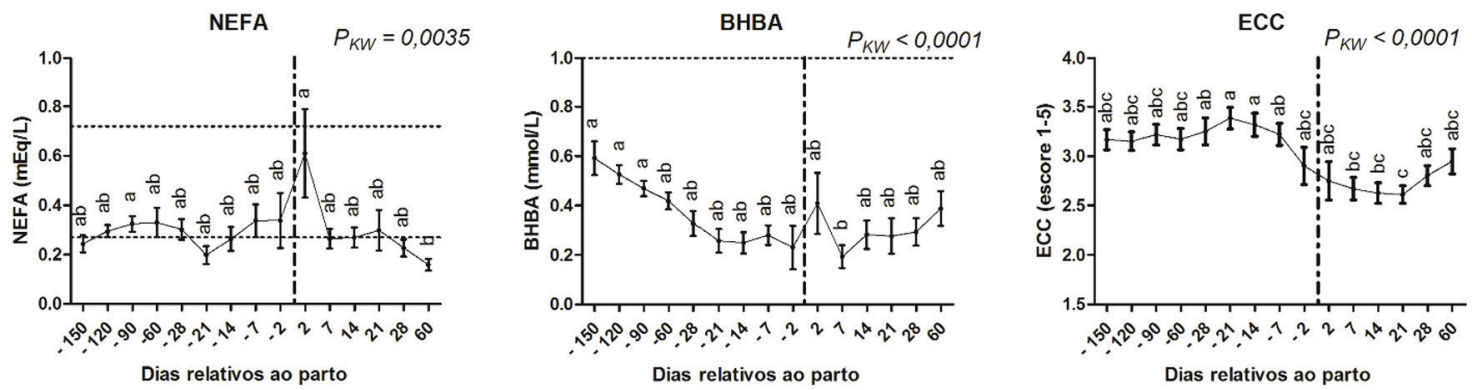

Fig.4. Perfil bioquímico energético de vacas Jersey multíparas clinicamente sadias, criadas em sistema semi-intensivo na região de Uberlândia, MG. (Nota: Letras diferentes entre os dias de avaliação indicam diferença estatística $(\mathrm{P}<0,05)$ pelo teste de Kruskall Wallis $\left(P_{\mathrm{KW}}\right)$. Vacas multíparas com produção média de $20 \mathrm{~kg}$ leite/dia. Linhas tracejadas representam o limite máximo do valor aceito para as variáveis conforme Ospina et al. (2010a) para NEFA $(<0,27 \mathrm{mEq} / \mathrm{L}$ no pré-parto e $<0,72 \mathrm{mEq} / \mathrm{L}$ no pós-parto) e BHBA $(<1,0 \mathrm{mg} / \mathrm{dL}$ no pós-parto).) 
Cálcio

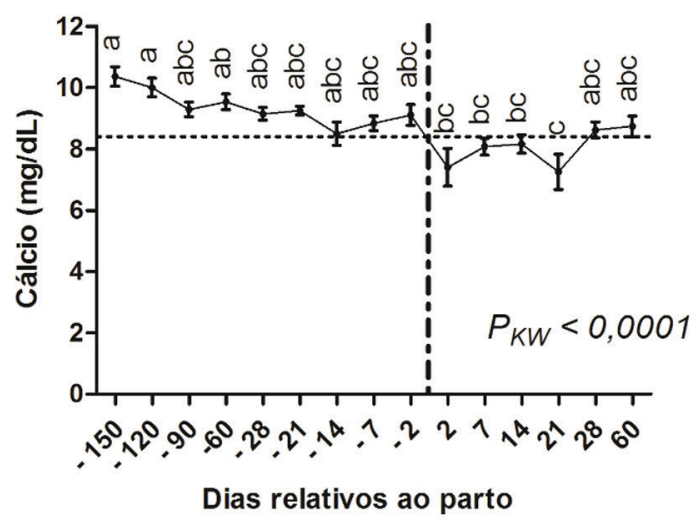

Magnésio

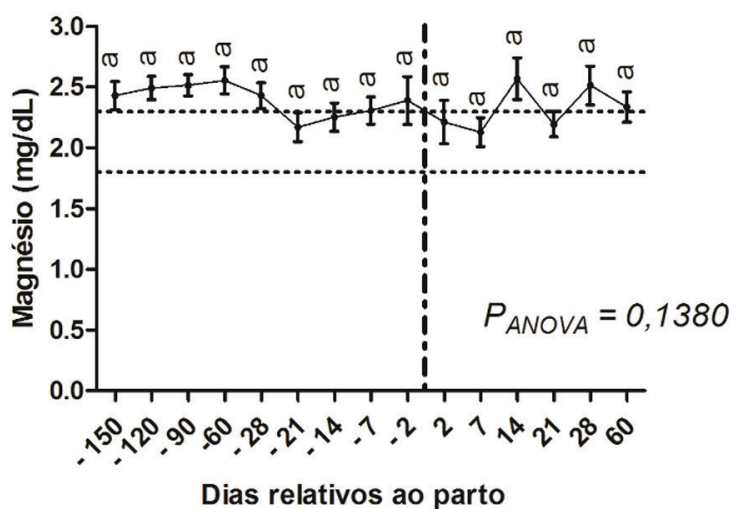

Fósforo

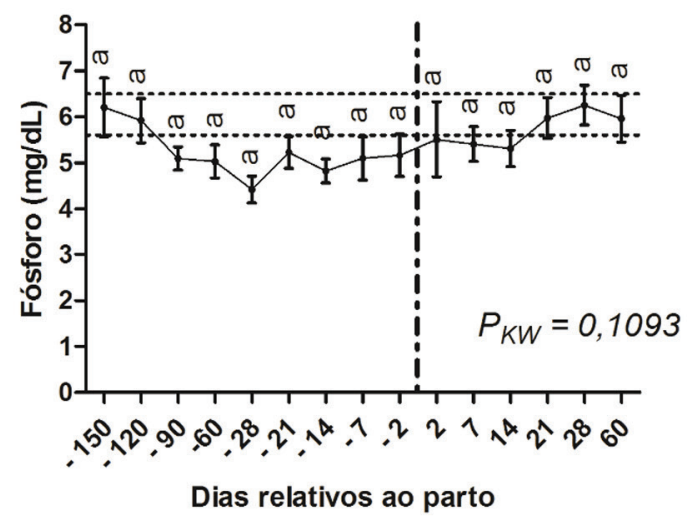

Relação cálcio/fósforo

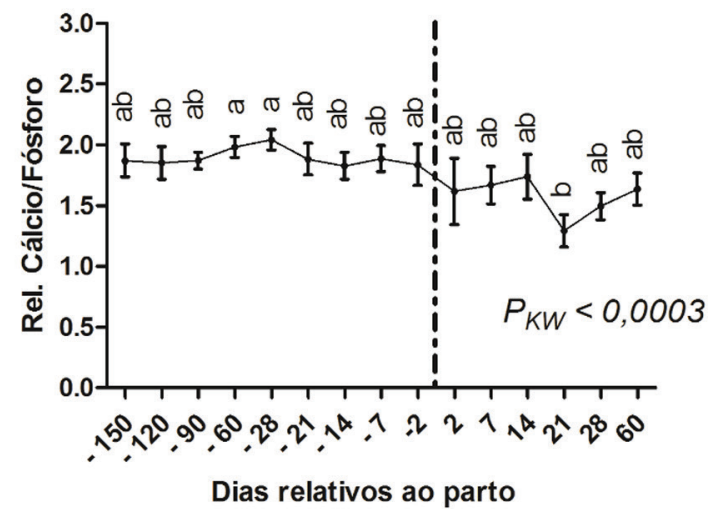

Fig.5. Perfil bioquímico sérico mineral de vacas Jersey multíparas clinicamente sadias, criadas em sistema semi-intensivo na região de Uberlândia, MG. (Nota: Letras diferentes entre os dias de avaliação indicam diferença estatística $(\mathrm{P}<0,05)$ pelo teste de Kruskall Wallis $\left(P_{\mathrm{KW}}\right)$ ou análise de variância $\left(\mathrm{P}_{\mathrm{ANOVA}}\right)$. Vacas multíparas com produção média de $20 \mathrm{~kg}$ leite/dia. Linhas tracejadas representam os limites dos valores de referência adotados conforme Chapinal et al. (2012) para cálcio (>8,4mg/dL) e segundo Smith (2009) para fósforo (5,6-6,5mg/dL) e magnésio (1,8-2,3mg/d|L).)

no pré-parto, com os valores das globulinas variando entre $5,51 \pm 2,31 \mathrm{~g} / \mathrm{dL}$ e $7,6 \pm 0,67 \mathrm{~g} / \mathrm{dL}$, sugere que esses achados possam estar relacionados com algum fator regional, ainda não identificado pelos autores, uma vez que, em outra pesquisa realizada na região de Uberlândia, MG, durante o mesmo período, com 34 vacas mestiças leiteiras de composição genética variando entre $1 / 4,1 / 2,5 / 8$ e $3 / 4$ Holandês/ Gir, observou-se comportamento semelhante de proteínas totais $(8,1 \pm 0,9 \mathrm{~g} / \mathrm{dL})$ e globulinas $(5,5 \pm 1,1 \mathrm{~g} / \mathrm{dL})$ nos dois meses anteriores ao parto (Daibert et al. 2016).

Não foi encontrado na literatura consultada suporte para explicar a hiperproteinemia por hiperglobulinemia ocorrida durante o pré-parto desses animais, especificamente para a raça Jersey (Katoh 2002, Gregory et al. 2004, Souza et al. 2004, Huzzey et al. 2009) e nem para a raça Holandesa (Souza et al. 2010, Saut et al. 2014). A origem desta hiperglobulinemia não foi objetivo desta pesquisa, no entanto, é importante que futuros trabalhos identifiquem qual a fração de globulinas que estaria aumentada, para que então se consiga chegar às causas para tal acontecimento repetido nos animais da região estudada.

A redução dos níveis proteicos e de globulinas observadas no pré-parto, não levou ao quadro transitório de hipoglobulinemia, fato descrito frequentemente nas pesquisas com animais no periparto (Gonçalves \& Kozicki 1997, Feitosa \& Birgel 2000, Saut et al. 2014) devido à mobilização das gamaglobulinas para formação de colostro na glândula mamária.

A baixa concentração de albumina concordou com os achados de Birgel Junior et al. (2003) que trabalharam com vacas Holandesas no terço final de gestação. No pós-parto esta hipoalbuminemia persistiu e, apesar do aumento verificado, manteve-se no limite inferior de referência (Smith 2009). Pesquisas têm apontado sugestões para essa hipoalbuminemia, que poderia ser atribuída a uma disfunção hepática ocorrida no pré-parto ou em função da ingestão de alimentos reduzida nessa fase, que pudesse inibir a síntese proteica (Gonçalves \& Kozincki 1997, Souza et al. 2008).

0 perfil bioquímico hepático permitiu excluir a possibilidade da hipoalbuminemia ser decorrente da redução na síntese hepática, pois esses animais não passaram por um quadro de lesões hepáticas, uma vez que os teores de AST e GGT se mantiveram dentro dos padrões de referência propostos por Smith (2009), de 43-127 UI/L e 15-39 UI/L, respectivamente. Estes resultados concordaram com Souza et al. (2010), que verificaram baixos níveis de albumina em vacas Holandesas, sem a influência do metabolismo hepático. Além disso, não se acredita que quadros de parasitose 
Quadro 1. Intervalo de confiança para cada parâmetro bioquímico avaliado de vacas Jersey clinicamente saudáveis, criadas em sistema semi-intensivo na região de Uberlândia, MG

\begin{tabular}{|c|c|c|c|c|c|c|c|c|}
\hline & \multicolumn{8}{|c|}{ Periparto (Dias relativos ao parto) } \\
\hline & -21 & -14 & -7 & -2 & 2 & 7 & 14 & 21 \\
\hline PT & $7,87-9,09$ & $7,44-9,38$ & $7,05-9,27$ & $5,44-8,92$ & $6,45-8,38$ & $7,34-8,57$ & $7,36-8,99$ & $7,7-8,97$ \\
\hline Alb. & $2,49-3,38$ & $2,4-3,0$ & $2,45-2,84$ & $2,51-3,24$ & $2,4-3,17$ & $2,65-3,47$ & $2,55-3,47$ & $2,55-3,16$ \\
\hline Glob. & $4,85-6,25$ & $4,69-6,72$ & $4,37-6,67$ & $2,16-6,42$ & $3,71-5,55$ & $4,25-5,55$ & $4,3-6,03$ & $4,93-6,03$ \\
\hline Rel. a/g & $0,42-0,58$ & $0,4-0,66$ & $0,45-0,64$ & $0,38-1,0$ & $0,46-0,81$ & $0,49-0,66$ & $0,46-0,64$ & $0,47-0,61$ \\
\hline Ureia & $23,17-39,15$ & $26,54-40,24$ & $27,60-39,88$ & $20,83-34,44$ & $34,75-44,82$ & $32,33-40,59$ & $30,45-43,93$ & $35,44-53,06$ \\
\hline Creat & $0,66-0,88$ & $0,70-0,87$ & $0,71-0,91$ & $0,65-0,93$ & $0,55-1,10$ & $0,63-0,78$ & $0,60-0,78$ & $0,62-0,75$ \\
\hline AST & $67,82-88,98$ & $73,92-101,4$ & $69,67-92,77$ & $58,84-92,77$ & $80,06-93,69$ & $71,00-96,80$ & $74,15-93,22$ & $72,53-91,26$ \\
\hline GGT & $12,03-21,30$ & $12,96-21,30$ & $13,14-20,33$ & $9,12-30,54$ & $11,60-20,70$ & $15,04-20,67$ & $13,32-19,49$ & $15,49-22,46$ \\
\hline CK & $152,8-812,4$ & $280,5-933,4$ & $161,8-1307$ & $109,3-647,6$ & $196,1-468,1$ & $242,0-709,4$ & $211,3-781,6$ & $171,0-338,6$ \\
\hline NEFA & $0,12-0,28$ & $0,16-0,37$ & $0,20-0,48$ & $0,05-0,63$ & $0,19-1,03$ & $0,18-0,35$ & $0,18-0,36$ & $0,13-0,47$ \\
\hline BHBA & $0,16-0,36$ & $0,16-0,34$ & $0,20-0,36$ & $0,01-0,46$ & $0,12-0,70$ & $0,10-0,29$ & $0,16-0,40$ & $0,12-0,43$ \\
\hline Col. & $65,74-96,93$ & $49,37-113,3$ & $52,04-85,14$ & $42,12-95,54$ & $59,81-90,99$ & $70,91-89,19$ & $78,58-105,6$ & $101,6-137,7$ \\
\hline Trig. & $10,98-24,75$ & $13,63-27,57$ & $12,20-34,59$ & $12,87-28,63$ & $2,34-35,46$ & $9,70-16,54$ & $11,13-30,78$ & $11,39-21,14$ \\
\hline HDL & $41,11-55,29$ & $35,42-67,91$ & $39,31-50,44$ & $25,53-62,80$ & $31,88-65,52$ & $47,82-64,58$ & $51,80-86,48$ & $65,30-87,97$ \\
\hline LDL & $12,33-51,62$ & $0,38-74,50$ & $9,61-47,16$ & $11,02-33,54$ & $12,45-33,39$ & $19,02-33,49$ & $16,82-39,23$ & $23,05-61,64$ \\
\hline VLDL & $2,20-4,95$ & $2,73-5,51$ & $2,23-6,58$ & 2,57- 5,67 & 1,34 - 3,53 & 1,55 - 8,12 & $2,15-6,68$ & $2,28-4,23$ \\
\hline $\mathrm{Ca}$ & $8,94-9,57$ & $7,70-9,30$ & $8,34-9,34$ & $8,25-9,97$ & $5,95-8,86$ & $7,5-8,66$ & $7,52-8,81$ & $6,04-8,47$ \\
\hline $\mathrm{P}$ & $4,48-5,97$ & $4,26-5,3$ & $4,10-6,09$ & $3,98-6,35$ & $3,58-7,44$ & $4,62-6,20$ & $4,48-6,14$ & $5,05-6,90$ \\
\hline $\mathrm{Mg}$ & $1,91-4,43$ & $2,0-22,5$ & $2,07-2,54$ & $1,89-2,89$ & $1,79-2,64$ & $1,88-2,38$ & $2,21-2,93$ & $1,98-2,41$ \\
\hline Rel. Ca/P & $1,60-2,16$ & $1,59-2,06$ & $1,66-2,11$ & $1,40-2,27$ & $0,97-2,26$ & $1,34-1,99$ & $1,35-2,12$ & $1,01-1,57$ \\
\hline
\end{tabular}

sejam responsáveis por essa redução no teor de albumina, uma vez que o rebanho estudado passou por um controle sanitário rigoroso.

Outro fator pouco provável, que pudesse justificar a hipoalbuminemia, foi a nutrição fornecida nesta fase, pois dietas com deficiência no aporte de energia e nutrientes poderia restringir a síntese hepática de proteínas. Além disso, foi constatado que a condição corporal dos animais $(E C C=3,17+0,11)$ foi condizente com a fase (Sartori \& Guardieiro 2010) e que a dieta fornecida aos animais foi formulada conforme as exigências do Nutrient Requirements of Dairy Cattle (NRC) (Council 2001). Os níveis de ureia apresentaram-se dentro dos valores de referência (23$58 \mathrm{mg} / \mathrm{dL}$ ) propostos por David Eckersall (2008), fato que demonstrou que o aporte proteico da dieta foi satisfatório.

Ressalta-se que todos os animais, no exame clínico proposto, eram clinicamente saudáveis, portanto, o fato de a albumina ser considerada uma proteína de fase aguda negativa nos processos inflamatórios agudos (Souza et al. 2010, Saut et al. 2014) talvez não tenha uma importância tão significativa nestes animais, durante o período avaliado. Portanto, após a exclusão de vários fatores que poderiam interferir na concentração de albumina reduzida no periparto, os autores consideram que estes valores encontrados possam ser normais da raça Jersey criada em clima tropical, contribuindo dessa forma na produção de novos valores de referência.

0 perfil sérico de colesterol manteve-se dentro dos valores esperados (Smith 2009), com sua redução no pré-parto devido à mobilização de gordura pela glândula mamária para a produção do colostro que, a partir do reestabelecimento da ingestão de alimentos, com menor exigência de gordura no leite, volta a assumir valores cres-
Quadro 2. Índices reprodutivos de vacas Jersey multíparas clinicamente sadias, criadas em sistema semi-intensivo na região de Uberlândia, MG

\begin{tabular}{lc}
\hline Número de animais (n) & 21 \\
Primeiro serviço (dias) & $45+6,2$ \\
Prenhez na 1o IA (\%) & 45 \\
Número de IA/prenhez & $1,95+0,2$ \\
Dias em aberto (mediana) & 58,5 \\
Dias em aberto (média) & $80,1+14,29$
\end{tabular}

centes no puerpério, conforme Pogliani (2006) e González et al. (2014).

A concentração sérica de triglicerídeos, sintetizados principalmente no fígado, tecido adiposo, glândula mamária e intestino delgado (Bruss 2008) esteve acima dos valores de referência de $0-14 \mathrm{mg} / \mathrm{dL}$ proposto por Smith (2009) sem alteração das enzimas hepáticas (AST e GGT), portanto, apesar de aumentado não interferiu na função hepática. Este fato tem sido relatado em trabalhos regionais, como o de Souza et al. (2010) que obtiveram índices de $17,83 \pm 5,37 \mathrm{mg} / \mathrm{dL}$ para vacas Holandesas até $10 \mathrm{DPP}$ com puerpério fisiológico.

O BEN foi observado em todas as vacas $(n=21)$ ao menos em um momento do periparto, sendo que $66,7 \%(14 / 21)$ apresentaram no pré-parto e $85,7 \%(18 / 21)$ no pós-parto e, discordaram de Dubuc et al. (2010), Ospina et al. (2010), Chapinal et al. (2011, 2012) e Roberts et al. (2012), que descrevem o BEN como fator de risco para a ocorrência de enfermidades puerperais e baixo desempenho reprodutivo.

0 fato de $100 \%$ das vacas clinicamente sadias terem passado por BEN no periparto e não terem prejuízo no desempenho reprodutivo, sugere que esta raça possa suportar os níveis de NEFA e BHBA estabelecidos como fatores de risco para doenças puerperais e interferência no desem- 
penho reprodutivo. Os autores propõem que talvez os valores críticos estabelecidos em animais criados no hemisfério norte não se apliquem à realidade dos animais deste experimento, havendo a necessidade de pesquisas futuras para o estabelecimento de valores de referência nacionais, apesar de esta inferência ser feita com uma amostragem baixa.

Como não foram encontrados na literatura compilada, sobretudo a nacional, estudos que citassem a incidência do BEN com base nos índices de NEFA e BHBA em vacas Jersey no periparto, este trabalho é pioneiro em disponibilizar dados que possam auxiliar na determinação de valores de referência que caracterizem esta fase, uma vez que, devido ao avanço das tecnologias empregadas no campo, existe demanda crescente de conhecimento acerca do padrão metabólico apresentado pelos animais escolhidos.

É importante ressaltar que 57,14\% (12/21) das vacas no pré-parto e 80,95\% (17/21) no pós-parto apresentaram valores de cálcio inferiores $(\mathrm{Ca}<8,4 \mathrm{mg} / \mathrm{dL}$ ) ao determinado por Chapinal et al. (2011) e Chapinal et al. (2012) como fator de risco para hipocalcemia subclínica, menor chance de prenhez à primeira IA e deslocamento de abomaso. De acordo com González \& Silva (2006) e González et al. (2014), essa hipocalcemia subclínica pode ser explicada pelo fato de vacas Jersey apresentarem população reduzida de receptores para a vitamina $\mathrm{D}_{3}$ (1,25-Dihidroxi-colocalciferol) no epitélio intestinal, que por sua vez, tem a importante função de ativar as proteínas ligantes de cálcio para sua absorção, bem como, ativar a ação da bomba de cálcio (Ca-ATPase), responsável por disponibilizá-lo para a corrente circulatória. No entanto, o fato de se observar valores de albumina próximos do limite mínimo de referência, poderia ser uma das justificativas dos valores baixos de cálcio, já que cerca de $45 \%$ do cálcio plasmático está ligado à albumina (González 2000).

Apesar de os animais aqui referidos apresentarem níveis críticos de cálcio no pós-parto, a não ocorrência de enfermidades puerperais pode ser devido ao fato de os níveis de NEFA não terem sido suficientes para promover tais desordens, como o descrito por Ospina et al. (2010) que correlaciona estas duas variáveis. Além disso, assim como já descrito para os valores de albumina, NEFA e BHBA, os autores veem a necessidade de se rever estes valores de cálcio para vacas Jersey criadas em climas tropicais. 0 mesmo deve ser considerado para os níveis de fósforo que foram encontrados abaixo dos valores de referência $(5,6-6,5 \mathrm{mg} /$ dL) (Smith 2009) no periparto.

Estes valores de fósforo poderiam estar diminuídos nesta fase devido à redução na ingestão de alimentos e à drenagem desse mineral para a glândula mamária, o que segundo González \& Scheffer (2002), González \& Silva (2006), González et al. (2014), poderia levar à sintomas semelhantes àqueles apresentados nos quadros de hipocalcemia, mas que não foi observado nesse grupo de animais.

Os resultados encontrados nesta pesquisa auxiliarão na formação de valores de referência para o perfil metabólico de vacas Jersey, uma vez que foram tomados os devidos cuidados para a não inclusão de animais que apresentassem qualquer alteração clínica e/ou metabólica durante todo o período avaliado, utilizando apenas aqueles clinicamente saudáveis, o que pode ser comprovado pela eficiência reprodutiva desses animais que se adequa aos índices adotados como padrão para vacas leiteiras segundo Alvarez et al. (2011).

\section{CONCLUSÕES}

Vacas da raça Jersey apresentam perfil bioquímico caracterizado por hiperglobulinemia durante o periparto, além de níveis elevados de triglicérides e colesterol, entretanto, sem comprometimento da função hepática.

0 balanço energético negativo e hipocalcemia subclínica não interferiram na ocorrência de doenças no periparto e desempenho reprodutivo de vacas Jersey criadas em condições de clima e manejo locais.

Agradecimentos.- Os autores agradecem à Fundação de Amparo à Pesquisa do Estado de Minas Gerais (FAPEMIG) e ao Conselho Nacional de Desenvolvimento Científico e Tecnológico (CNPq) pelo apoio financeiro para a execução do trabalho, à Coordenação de Aperfeiçoamento de Pessoal de Nível Superior (CAPES) pela bolsa de estudos concedida e ao proprietário e funcionários da Fazenda Tira Leite.

\section{REFERÊNCIAS}

Aikman P.C., Reynolds C.K. \& Beever D.E. 2008. Deit, digestibility, rate of passage, and eating and rumination behavior of Jersey and Holstein cows. Am. Dairy Sci. Assoc. 91:1103-1113.

Alvarez R.H., Niciura R., Santos R.M. \& Franceschini P.H. 2011. Glossary of bovine physiology, pathology and reproductive performance terms. Cont. Educ.Vet. Med. Zootec. CRMV-SP 9:12-23.

Birgel Junior E.B., Neves F.S., Salvatore L.C.A., Mirandola R.M.S., Távora J.P.F. \& Birgel E.H. 2003. Gestation and puerperium influence over the hepatic function of holstein cows. Ars Vet. 19:172-178.

Bruss M.L. 2008. Lipids and ketones, p.81-115. In: Kaneko J.J., Harvey J.W. \& Bruss M.L. (Eds), Clinical Biochemistry of Domestic Animals. 6th ed. Academic Press, San Diego.

Chapinal N., Carson M., Duffield T.F., Capel M., Godden S., Overton M., Santos J.E.P. \& LeBlanc S.J. 2011. The association of serum metabolites with clinical disease during the transition period. J. Dairy Sci. 94:4897-4903.

Chapinal N., LeBlanc S.J., Carson M.E., Leslie K.E., Godden S., Capel M., Santos J.E.P., Overton M.W. \& Duffield T.F. 2012. Herd-level association of serum metabolites in the transition period with disease, milk production, and early lactation reproductive performance. J. Dairy Sci. 95:5676-5682.

Council N.R. 2001. Nutrient Requirements of Dairy Cattle. Disponível em <http://www.nap.edu/catalog/9825/nutrient-requirements-of-dairy-cattle-seventh-revised-edition-2001> Acesso em 15 dez. 2015.

Daibert E., Fagundes N.S., Rezende A.L., Alvarenga P.B., Krüger B.C., Santos R.M., Mundim A.V. \& Saut J.P.E. 2016. Influência do balanço energético negativo sobre enfermidades uterinas e perfil metabólico de vacas leiteiras mestiças no periparto. Universidade Federal de Uberlândia, MG. Dados não publicados.

David Eckersall P. 2008. Proteins, proteomics, and the dysproteinemias, p.117-155. In: Kaneko J.J., Harvey J.W. \& Bruss M.L. (Eds), Clinical Biochemistry of Domestic Animals. 6th ed. Academic Press, San Diego.

Dubuc J., Dufield T.F., Leslie K.E., Walton J.S. \& LeBlanc S.J. 2010. Risk factors for postpartum uterine diseases in dairy cows. J. Dairy Sci. 93:57645771.

Edmonson A.J., lean I.J., Weaver L.D., Farver T. \& Webster G. 1989. A Body condition scoring chart for holstein dairy cows. J. Dairy Sci. 72:68-78.

Feitosa F.L.F. 2014. Semiologia Veterinária: a arte do diagnóstico. 3aa ed. Roca, São Paulo. 735p.

Feitosa F.L.F. \& Birgel E.H. 2000. Variation of G and M immunoglobulins, total protein and eletrophoretic fractions and activity of gama glutamyl- 
transferase in Holstein cow blood sera before and after parturition. Arq. Bras. Med. Vet. Zootec. 52:111-116.

Ferreira A.D.M. 2010. Reprodução da Fêmea Bovina: fisiologia aplicada e problemas mais comuns (causas e tratamentos). 1aㅡ edição do autor, Juiz de Fora, MG. 420p.

Friedewald W.T., Levy R.I. \& Redrickson D.S. 1972. Estimation of the concentration of low-density lipoprotein cholesterol in plasma, without use of the preparative ultracentrifuge. Clin. Chem. 18:499-502.

Giffoni S.N. \& Rosa R. 2007. Mapeamento dos remanescentes da cobertura vegetal natural do Município de Uberlândia no ano de 2002. Anais XIII Simpósio Brasileiro de Sensoreamento Remoto, Florianópolis, SC, p.1679-1686,

Gilbert R.O., Shin S.T., Guard C.L., Erb H.N. \& Frajblat M. 2005. Prevalence of endometritis and its effects on reproductive performance of dairy cows. Theriogenology 64:1879-1888.

Gonçalves D. \& Kozicki L.E. 1997. Biochemical and immunological profiles during the parturition period in dairy cows with and without placental retention. Braz. J. Vet. Res. Anim. Sci. 34:364-370.

González F.H.D. 2000. Indicadores sanguíneos do metabolismo mineral em ruminantes, p.31-51. In: González F.H.D., Barcellos J., Patiño H.O. \& Ribeiro L.A. (Eds), Perfil Metabólico em Ruminantes: seu uso em nutrição e doenças nutricionais. Editora da UFRGS, Porto Alegre, RS.

González F.H.D. \& Scheffer J.F.S. 2002. Perfil sanguíneo: ferramenta de análise clínica, metabólica e nutricional, p.5-17. In: Avaliação metabólico-nutricional de vacas leiteiras por meio de fluídos corporais (sangue, leite e urina). Anais do curso realizado no 29을 Congresso Nacional de Medicina Veterinária. Gramado, RS. 72p.

González F.H.D. \& Silva S.C. 2006. Introdução à Bioquímica Clínica Veterinária. $2^{\mathrm{a}}$ ed. Editora da UFRGS, Porto Alegre, RS. 358p.

González F.H.D., Corrêa M.N. \& Silva S.C.D. 2014. Transtornos Metabólicos nos Animais Domésticos. $2^{\text {a }}$ ed. Editora da UFRGS, Porto Alegre, RS. $337 p$.

Gregory L., Birgel Junior E.B., D’Angelino J.L., Benesi F.J., Araújo W.P. \& Birgel E.B. 2004. Valores de referência dos teores séricos da ureia e creatinina em bovinos da raça jersey criados em São Paulo. Influência dos fatores etários, sexuais e da infecção pelo vírus da leucose dos bovinos. Arqs Inst. Biológico, São Paulo, 7:339-345.

Huzzey J.M., Duffield T.F., LeBlanc S.J., Vieira D.M., Weary D.M. \& Keyserlingk M.A.G. 2009. Haptoglobin as an early indicator of metritis. J. Dairy Sci. 92:621-625.

Kasimanickam R., Duffield T.F., Foster R.A., Gartley C.J., Leslie K.E., Walton J.S. \& Johnson W.H. 2005. A comparison of the cytobrush and uterine lavage techniques to evaluate endometrial cytology in clinically normal postpartum dairy cows. Can. Vet. J. 46:255-259.

Katoh N. 2002. Relevance of apolipoproteins in the development of fatty liver and fatty liver-related peripartum diseases in dairy cows. J. Vet. Med. Sci. 64:293-307.

Kaufmann T.B., Drillich M., Tenhagen B.A., Forderung D. \& Heuwieser W. 2009. Prevalence of bovine subclinical endometritis $4 \mathrm{~h}$ after insemination and its effects on first service conception rate. Theriogenology 71:385-391.

Martins C.F.G., Oliveira P.M., Nasciutti N.R., Barbosa V.M., Ferreira M.B., Maturana Filho M., Santos R.M., Mota F.C.D. \& Saut J.P.E. 2013. Gynecological and cytological evaluation in Holstein cows with more than three repetitions of estrus. Semina, Ciênc. Agrárias 34:3787-3794.

Oliveira R.S.B.R., Moura A.R.F., Pádua M.F.S., Barbon I.B., Silva M.E.M., Santos R.M., Mundim A.V. \& Saut J.P.E. 2014. Metabolic profile in crossbred dairy cows with low body condition score in the peripartum period. Pesq. Vet. Bras. 34:362-368.

Ospina P.A., Nydam D.V., Stokol T. \& Overton T.R. 2010. Association between the proportion of sampled transition cows with increased nonesterified fatty acids and beta-hydroxybutyrate and disease incidence, pregnancy rate, and milk production at the herd level. J. Dairy Sci. 93:3595-601.

Peixoto L.A.O., Osório M.T.M., Osório J.C.S., Nörnberg J.L. \& Pazini M. 2010. Reproductive performance and blood metabolites from ile de france ewes fed organic or common salt in the breeding season. Revta Bras. Zootec. 39:191-197.

Pogliani F.C. 2006. Reference values and influence of age, gender and gestation on the lipid profile of Holstein cattle, bred in the state of São Paulo. Dissertação de Mestrado em Clínica Veterinária, Faculdade de Medicina Veterinária e Zootecnia, Universidade de São Paulo, São Paulo, SP. $136 \mathrm{p}$.

Prendiville R., Pierce K.M. \& Buckley F. 2009. An evaluation of production efficiencies among lactating Holstein-Friesian, Jersey, and Jersey x Holstein-Friesian cows at pasture. Am. Dairy Sci. Assoc. 92:6176-6185.

Rezende E.V. 2013. Incidence of retained placenta and the consequences on milk produc.tion and reproductive efficiency of holstein cows. Dissertação de Mestrado em Ciências Veterinárias, Faculdade de Medicina Veterinária, Universidade Federal de Uberlândia, Uberlândia, MG. 41p.

Roberts T., Chapinal N., LeBlanc S.J., Kelton D.F., Dubuc J. \& Duffield T.F. 2012. Metabolic parameters in transition cows as indicators for early-lactation culling risk. J. Dairy Sci. 95:3057-3063.

Rucker R.B., Fascetti A.J. \& Keen C.L. 2008. Trace minerals, p.663-693. In: Kaneko J.J., Harvey J.W. \& Bruss M.L. (Eds), Clinical Biochemistry of Domestic Animals. 6th ed. Academic Press, San Diego.

Santos A.B. \& Petronzio J.A.C. 2011. Mapeamento de uso e ocupação do solo do município de Uberlândia/MG utilizando técnicas de geoprocessamento. Anais XV Simpósio Brasileiro de Sensoriamento Remoto (SBSR), Curitiba, PR, p.6185.

Sartori R. \& Guardieiro M.M. 2010. Nutritional factors associated with reproduction in heifers and cows. Revta Bras. Zootec. 39:422-432.

Saut J.P.E., Miyashiro S.I., Raimondo R.F.S., Nunes M.T., Mori C.S., Fagliari J.J. \& Birgel Junior E.H. 2014. Retained placenta on the proteinogram of Holstein cows. Ciência Rural 44:1651-1657.

Sheldon I.M. 2004. The postpartum uterus. Vet. Clin. North Am., Food Anim. Pract. 20:569-591.

Sheldon I.M., Williams E.J., Miller A.N.A., Nash D.M. \& Herath S. 2008. Uterine diseases in cattle after parturition. Vet. J. 176:115-121.

Sheldon I.M., Cronin J., Goetze L., Donofrio G. \& Shuberth H.J. 2009. Defining postpartum uterine disease and the mechanisms of infection and immunity in the female reproductive tract in cattle. Biol. Reprod. 81:1025-1032.

Smith B.P. 2009. Large Animal Internal Medicine. 4th ed. Mosby Elsevier, Missouri. 1825p.

Souza R.M., Garcia N.A.C.R., Birgel D.B. \& Birgel Junior E.H. 2008. Influence of puerperium and post-puerperal phase on the hepatic function of health Holstein cows. Ciênc. Anim. Bras. 9:140-147.

Souza R.M., Birgel Junior E.H., Ayres M.C.C. \& Birgel E.H. 2004. Influence of breed factor on the hepatic function in Holstein and Jersey cattle. Braz. J. Vet. Res. Anim. Sci. 41:306-312.

Souza R.M., Yasuoka M.M., Leão D.A. \& Birgel Junior E.H. 2010. Relationship among hepatic function, lipid profile and endometrium inflammatory disorders (acute postpartum endometritis, placental retention and genital catarrhal discharges) in holstein cows. Ciênc. Anim. Bras. 11:880-887.

Williams E.J., Fischer D.P., Pfeiffer D.U., England G.C.W., Noakes D.E., Dobson H. \& Sheldon I.M. 2005. Clinical evaluation of postpartum vaginal mucus reflects uterine bacterial infection and the immune response in cattle. Theriogenology 63:102-117. 